\title{
Phased source arrays
}

C Tauchner* (Montanuniversität Leoben), F Bleibinhaus (Montanuniversität Leoben)

\section{Summary}

Quarries and open pit mines face challenges in areas with surrounding infrastructure. Unavoidable side effects of rock blasting like ground vibrations are subject to many legal restrictions. Mines use vastly different methods to predict vibrations, which are either based on trial-and-error, or on very simplified models which neglects complex subsurface properties and topography. The phased source array approach utilizes angle dependant wave interferometry to reduce blasting vibrations at certain sensitive targets. In order to compute vibration models a subsurface velocity model, obtained with a seismic tomography experiment, is needed. 


\section{Introduction}

Quarries and open pit mines face tremendous challenges in areas with dense settlement. Unavoidable side effects of rock blasting like ground vibrations, air blast and flying rocks are subject to many legal restrictions. Blasting factors that influence ground vibrations include drill hole diameter, charge weight, delay times, topography and geology (Ainalis 2017). State of the Art methods to predict vibrations are either based on trial-and-error, or on very simplified models which neglect site specific factors, such as viscoelastic subsurface properties and topography.

\section{Phased source arrays and velocity model}

The project focuses on reducing blasting vibrations at sensitive targets with phased source arrays. This method utilizes interferometry to produce negative interference at certain sensitive angles in respect to the line of blasts. Meaning that negative interference at certain sensitive targets can protect infrastructure in vicinity from blasting vibrations. The future objective is to introduce a new method which utilizes state-of-the-art seismic techniques of waveform modelling in combination with electronic delay detonators to predict ground vibrations at certain sensitive targets. In order to be able to compute vibration models with this new method a velocity model of the underground is needed. Therefor the first seismic traveltime tomography experiment at Mt. Erzberg mine in Austria has taken place in November 2016. The aim was to collect data for a velocity model which describes the underground reasonably well. Therefore 1253 -component-stations were set up in a $4 \mathrm{~km}^{2}$ large area, within and around the active mine. During the 3 weeks of experiment instalment 21 smaller seismic shots as well as 10 production blasts were recorded. The velocity model from this data which also includes topography information will be used for interferometric waveform modelling and further calculations for optimal blasting patterns.

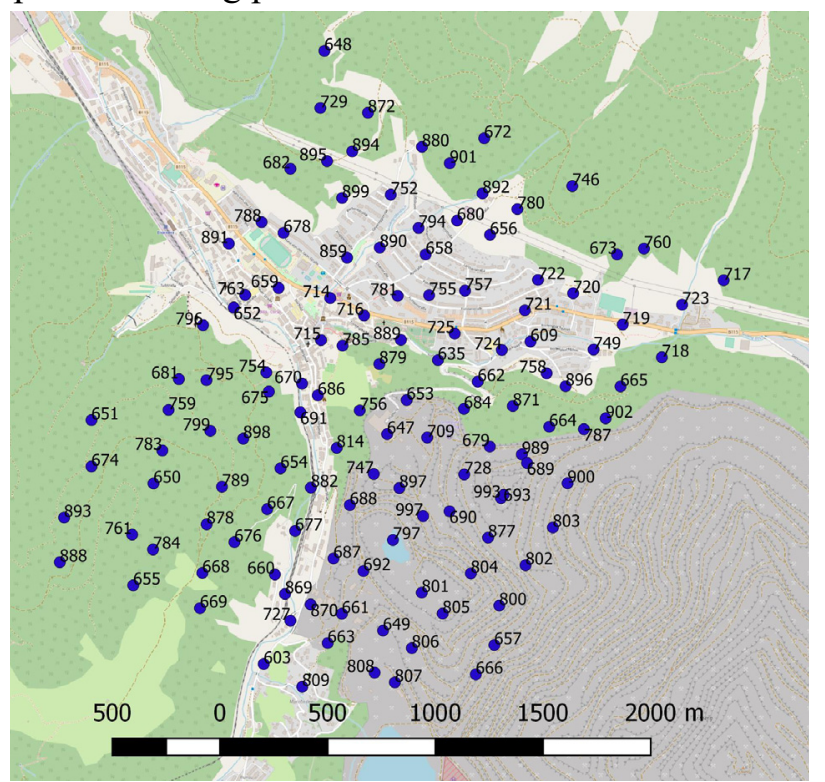

Figure 1 Deployment of 125 seismic stations at Mt. Erzberg

\section{Acknowledgement}

SLIM project has received funding from the European Union's Horizon 2020 research and innovation programme under grant agreement No: 730294.

\section{References}

Ainalis, D. et al., (2017) Modelling the Source of Blasting for the Numerical Simulation of Blast-induced Ground Vibrations: A Review. Rock Mech Rock Eng 50. S.171-193 